\title{
Is there a role for computational fluid dynamics (CFD) in neurovascular compression syndrome?
}

\author{
Prasad Temkar ${ }^{1}$, Yoko Kato ${ }^{2}$, Yasuhiro Yamada ${ }^{2}$, Ahmed Ansari ${ }^{2}$ \\ ${ }^{1}$ Apollo Institute of neurosciences, Apollo Speciality hospitals, Chennai, INDIA \\ ${ }^{2}$ Department of Neurosurgery, Fujita Health University, Banbuntane Hotokukai Hospital, \\ JAPAN
}

\begin{abstract}
Introduction - CFD uses complex algorithms to predict flow of fluids through a tube \& also predicts its impact on walls. It is increasingly being used for prediction of aneurysmal wall thickness, flow, wall shear stress and to decide clip strategy. This is an experimental study, which analyses use of CFD in neurovascular compression syndrome and probable future applications. We analysed CFD findings in 12 cases retrospectively to know fluid dynamics of vessels and its implications. Methodology - Twelve patients - 7 cases of trigeminal neuralgia and 5 of hemifacial spasm who were to be treated by MVD, were enrolled. CFD i.e. WSSv (wall shear stress vector), WSSm (magnitude), SL (stream line) and Pressure, was done in all prospectively. Results - CFD of superior cerebellar artery, anterior inferior cerebellar artery and vertebral artery was done in each case at the contact site and immediate pre + post contact location. Comparitively High WSSm correlated maximally at nerve vessel contact junction $(\mathrm{NVCj})$ and also high SL at the $\mathrm{NVCj}$ comparing with nerve vessel contact proximal (NVCp), WSSv showed wide variations and pressure change was in-conclusive. Conclusion - At contact site, the high WSSm\& SL might be a reliable indicator of a neurovascular conflict zone. To conclusively validate the findings we need large number of patients and control group.
\end{abstract}

Key words: Computational fluid dynamics, neuralgia, nerve vessel contact.

\section{Introduction}

Dandy was the first to report vascular compression of the trigeminal nerve, since then the concept of neurovascular compression syndrome for trigeminal neuralgia and hemifacial spasm (HFS) developed. (1) The pathophysiology being pulsatile compression of the offending artery over the affected nerve at the root entry zone(REZ). Microvascular decompression still remains the mainstay for treatment. Precise assessment of the complex nervevessel relationship at the root entry zone of the trigeminal nerve is useful for planning 
microvascular decompression in patients with idiopathic trigeminal neuralgia.2 Recently a fusion imaging technique for $3 \mathrm{D}$ MR cisternography and angiography by compositing a 3D MR cisternogram and a coregistered 3D MR angiogram in a single 3D image, has been described to provide a reliable assesment of the same. (3)

Computational Fluid Dynamics (CFD) uses complex algorithms to predict flow of fluids through a tube \& also predicts its impact on walls. It is increasingly being used for prediction of aneurysmal wall thickness, flow, wall shear stress and to decide clip strategy. (5) This is an experimental study, which we use of CFD to analyze the hemodynamics in offending arteries of neurovascular compression syndrome.

\section{Materials and Methods}

Data was collected retrospectively from 12 patients (7 TGN, 5 HFS; women, men; age range, $34-87$ years; mean, 63.9 years) with neurovascular compression syndromes (Table 1). The offending arteries as seen on preoperative imaging and intraoperative findings were (SCA, PICA, VA). In all cases MVD was done via the retrosigmoid approach.

All patients were imaged by means of 3D MR cisternography, 3D MR angiography (MRA) and 3D CT angiography (CTA). A 3D fusion image was created by superimposing the obtained images \& neurovascular conflict at the root entry zone was visualized. Surgical simulation images were prepared before surgery and were compared with the intraoperative surgical field image. CFD analysis done of contrast enhanced Multi Detector CTA images with post processing done on Ziostation 2 software. The offending vessel was isolated \& shape was reconstructed. CFD analysis was done using Hemoscope- v1.5(EBM, tokyo) and values for Wall shear stress(WSSm), Wall shear stress vector((WSSv), streamline flow velocity(SL), \& circumferential pressure was calculated. After confirmation intraoperatively, site of neurovascular conflict (NVCj), proximal (NVCp) and distal (NVCd) to it was marked in Hemoscope. The relative ratio's of NVCj to NCVp and NVCj to NVCd were calculated.

\section{Results}

The 3D fusion image created before surgery was compared with intraoperative locali-zation of compression at REZ. These respective zones were marked as $\mathrm{NVCj}$ (junction), NVCp (proximal) \& NVCd (distal). The values of WSSm, SL, PD are variable according to systolic and diastolic cardiac cycles6. Hence, their comparitive ratio's were used to remove the bias and equated to less than 1 , if low or more than 1 if high. If the comparision was relatively close then it was equated to one. Detailed results of each patient and their respective comparision values of WSSm, SL \& PD have been presented in table 2. The WSSv has been represented as presence (+) or absence (-) of change of linearity at a particular junction. The generalized patient population data on terms of age, sex, offending artery, side of symptoms, comorbidities \& drugs used have been presented in detail in Table 1 . 
WSSm comparing NVCj/NVCp was high $(>1)$ in 8 cases, 2 cases were almost equal, low in 2 cases; $\mathrm{NVCj} / \mathrm{NVCd}$ was high in 9 cases, low in 1 case, equal in case. SL comparing $\mathrm{NVCj} / \mathrm{NVCp}$ was high $(>1)$ in 9 cases, 3 cases were almost equal, never low; $\mathrm{NVCj} / \mathrm{NVCd}$ was high in 9 cases, low in 2 cases, equal in 1 cases. Pressure loss comparing $\mathrm{NVCj} / \mathrm{NVCp}$ was high $(>1)$ in 3 cases, 1 case was almost equal, low in 7 cases; NVCj/NVCd was high in 6 cases, low in 5 cases, equal in case. WSSv showed variation in direction at $\mathrm{NVCj}$ in 7 cases, at NVCp in 4 cases, at NVCd in 2 cases.
Table 1- Demographics of the affected patients in the study.

\begin{tabular}{|l|c|c|c|c|c|}
\hline $\begin{array}{l}\text { NVC } \\
\text { syndrome }\end{array}$ & side & age & sex & comorbids & artery \\
\hline TGN & LEFT & 73 & F & HTN,DM & SCA \\
\hline HFS & RIGHT & 40 & F & - & AICA \\
\hline TGN & LEFT & 70 & M & DM & SCA \\
\hline TGN & LEFT & 35 & F & HTN,DM & SCA \\
\hline TGN & RIGHT & 74 & M & HTN & SCA \\
\hline HFS & LEFT & 72 & F & DM & VA \\
\hline TGN & LEFT & 77 & F & HTN & SCA \\
\hline HFS & LEFT & 74 & F & - & AICA \\
\hline HFS & LEFT & 73 & M & - & AICA \\
\hline TGN & RIGHT & 66 & F & HTN & SCA \\
\hline TGN & LEFT & 61 & F & HTN,DM & SCA \\
\hline HFS & RIGHT & 47 & M & - & AICA \\
\hline
\end{tabular}

Table 2 - Summary of Patients Data and Comparision of Hemodynamic Charcteristics with CFD

\begin{tabular}{|c|c|c|c|c|c|c|}
\hline HFS & $>1$ & $>1$ & $>1$ & $>1$ & $<1$ & $<1$ \\
\hline TGN & 1 & $>1$ & 1 & $>1$ & $<1$ & $<1$ \\
\hline Lt TGN & $>1$ & $>1$ & $>1$ & $>1$ & $<1$ & $<1$ \\
\hline Rt TGN & $>1$ & $>1$ & $>1$ & $>1$ & $>1$ & $<1$ \\
\hline Lt HFS & $>1$ & $>1$ & $>1$ & $>1$ & $<1$ & $>1$ \\
\hline Lt TGN & $>1$ & $<1$ & $>1$ & $>1$ & $<1$ & $<1$ \\
\hline Lt HFS & 1 & $>1$ & 1 & $>1$ & $<1$ & $>1$ \\
\hline Lt HFS & $>1$ & $>1$ & 1 & 1 & $<1$ & $>1$ \\
\hline Rt TGN & & & $>1$ & $>1$ & 1 & $>1$ \\
\hline Lt TGN & $>1$ & $>1$ & $>1$ & $<1$ & & \\
\hline
\end{tabular}

TGN - trigeminal neuralgia, HFShemifacial spasms; NVCp- Proximal NVC region, NVCj- Junction NVC site, NVCdDistal NVC region, FV- Flow velocity, WSSm- Wall shear stress magnitude, WSSvWall shear stress vector, PD- Pressure loss.
As it is comparision study ratio's were averaged with following values $>1$ - high; $<1$ Low; 1 - both values approximately equal.

WSSv variations; (+) present, (-) absent 


\section{Representative cases}

Case 1:

A 64 years lady with no comorbidities complained of typical lancinating pain in the right V1 distribution, on carbamazepin (max dosage) for last 3 months without resolution of symptoms and depression. MRI showed neurovascular conflict at REZ of Vth nerve by superior cerebellar artery(SCA); She underwent transposition of the SCA with good relief of symptoms immediate post operatively.

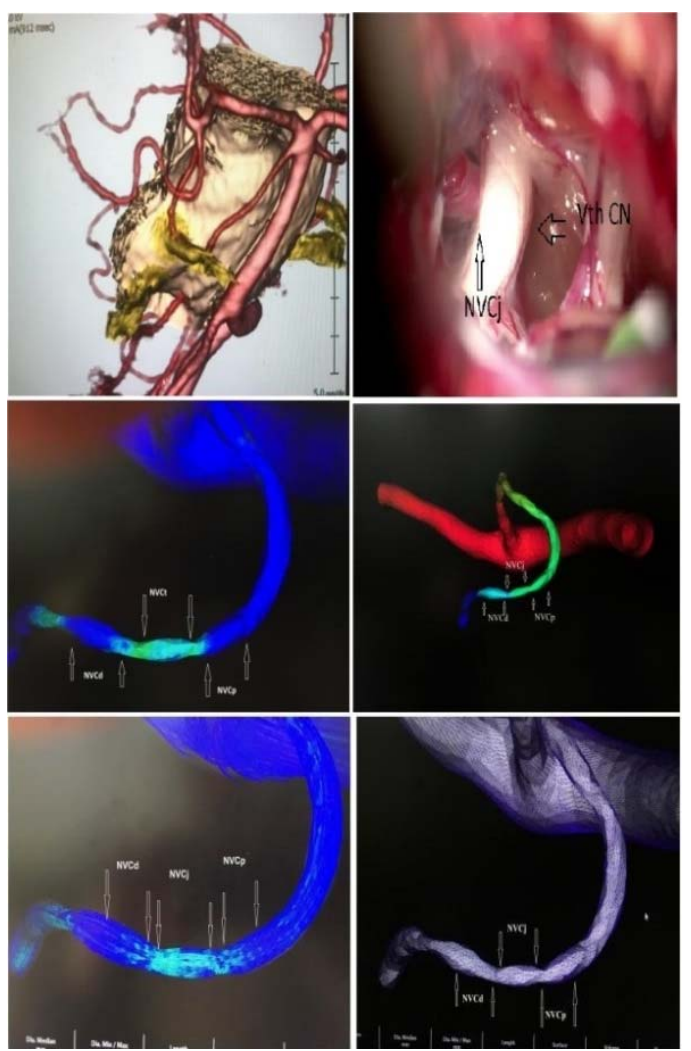

Figure 1- Clockwise from top: A) 3D CTA with 3D MRA recon.; B)Intraop view; C)WSSm; D)Pressure: E)Streamline flow velocity; F)WSSV
After superimposing 3D CTA over 3D MRA, the vessel was reconstructed on Ziostation. CFD analysis of the PICA with attention near the area of neurovascular conflict was done in VCFD of Hemosopev1.5. CFD analysis of the SCA with attention near the area of neurovascular conflict was done. WSSm was higher at NVCj than both NVCp or NVCd. Pressure was lower at NVCj compared to NVCp but higher than NVCd. SL also was higher at NVCj as compared to both NVCp\&NVCd. WSSv showed some variation in direction at $\mathrm{NVCj}$ site but was almost linear at NVCp\&NVCd. Patient had come for follow up after 6 months with good pain relief \& completely off medications.

\section{Case 2:}

A 77 year old hypertensive, diabetic lady presented with left sided persistant recurring hemifacial spasms which interfered with her way of life. She was on clonazepam for 6 months without any relief. MRI showed a tortuous anterior inferior cerebellar artery(AICA) compressing the 7 th nerve anteriorly and posteriorly. She was evaluated with 3D CTA, 3D MR cisternography and 3D MRA. She underwent transposition of PICA at first without relief and was re-operated with re transposition of AICA and teflon padding all around. 


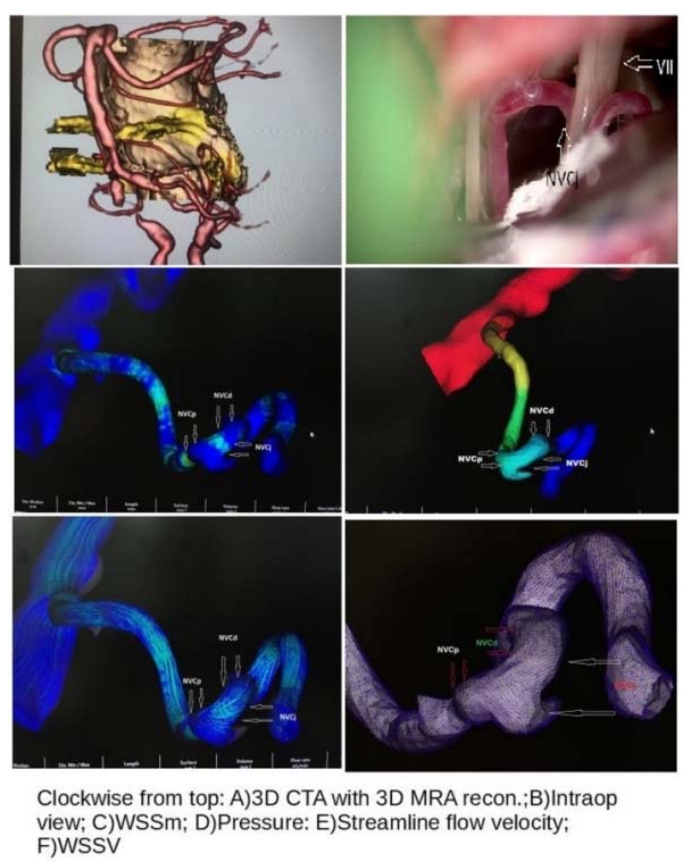

After superimposing 3D CTA over 3D MRA, the vessel was reconstructed on Ziostation. CFD analysis of the PICA with attention near the area of neurovascular conflict was done in VCFD of Hemosopev1.5.WSSm was higher at NVCj than both NVCp or NVCd. Pressure was lower at NVCj compared to NVCp but higher than NVCd. SL also was higher at NVCj as compared to both NVCp\&NVCd. WSSv showed some divergant vectors iatNVCj site but were almost linear at NVCp\&NVCd. Patient had come for follow up after 6 months with good pain relief \& completely off medications.

\section{Discussion}

Neurovascular conflict (compression) syndromes viz., HFS \&TGN, usually produce discomforting symptoms which prevent individuals from having a normal life. Microvascular decompression is the only permanent solution. They have a high recurrence rate if not treated correctly. Hence there is a need for precise evaluation of the conflict zone to plan course of surgery. The primary pathology is the nerve being compressed by a pulsatile artery and vice versa. Hence it is postulated that some hemodynamic changes would be seen at the site6. Recently role of CFD in analysing the growth of unruptured aneurysms has been studied extensively $[9,10]$. Also the perianeurysmal\& intra aneurysmal hemodynamics have been studied using CFD $\&$ increasingly being used in prediction of aneurysmal wall thickness, flow, wall shear stress and accordingly clip strategy is decided. $[7,8]$ Therefore the interest in analysing the hemodynamics of offending vessel at the site of compression.

CFD uses complex mathematical algorithms requiring high end computer softwares making it difficult to apply in daily use. It also provides a lot of CFD parameters, evaluation of analysis result and that the value is also not constant. Hence we used a dedicated software Hemoscope-v1.5 to analyse the blood flow at the site of neurovascular conflict. From the medical images information (DICOM data) of the patient, the blood vessel shape in $3 \mathrm{D}$ is created with Ziostation 2. Next, in Hemoscope-v1.5,vessel module the vessel inlet ,NVCj is marked \& with VCFD module analysis module FV, SL, WP, WSSv, were obtained. 
Wall shear stress (WSSm) results from friction between blood flow and the vessel wall, creating a tangential force exerted on the wall. High WSSm meant that flow is fast near endothelium \& low WSSm meant slow flow near endothelium - usually seen proximal to stenotic areas leads to plaque formation. In our series also we concur with the above as ratio of NVCj/NVCp was high in most of our findings. Sato et al have also reported similar findings in their study on hemifacial spasm.6WSSv indicates the direction of shear stress at wall. Usually parallel vectors are seen in straight vessel.While convergent and divergent vectors are found at turn or branching or Stenotic (Thick) or dilated areas (Thin). Change in the direction of vector in straight vessel could be due to external factors like compression/traction of vessel. We noted some change in direction of vectors at the NVCj in many cases but sometimes also at NVCp or NCVd. So this evidence was not substantial to qualify the above. Pressure is the force of blood column on the arterial wall circumferentially leading to stretching/stress on all wall layers. At higher pressures the intima is affected leading to remodeling of vessel wall or atherosclerotic changes. In our series pressurewascomparitively lower at NVCj but did not show significant $p$ value to confirm the association. Streamline flow velocity(SL) is the measurement of blood flow velocity at a given point. At low velocity the flow was streamline or linear, whereas at turns or stenotic areas it showed turbulent flow with high velocity. In our series, we have a good correlation of high SL at NVCj compared to NVCp. We think it may be due to the stenosis caused by compression, and also where it was equal may be due to remodeling of the intima in some cases having long duration of symptoms.

\section{Limitations of the study}

The CFD analysis was done at a cardiac cycle common to a single study but could not be generalized in every case as done by Sato et al.; butcomparision was made within one study to remove the bias. The compressed vessels are sometimes very thin and may not be able to be reconstructed correctly. Also overlying vessels or bone may add artifacts while recreating vessel shape. The software Hemoscope-v1.5(tokyo) was primarily made to asses perianeurysmal environment and we have applied it to smaller arteries. 6

\section{Conclusions}

Comparatively high WSSm and high SL at NVCj to NVCp correlates well with area of neurovascular conflict. Due to lack of sufficiently large number of cases, its validity remains a question. But this study certainly provides hope that the role of CFD in neurovascular syndromes is certainly the future. Future applications may involve use of CFD analysis postoperatively after complete resolution or recurrence of symptoms and compare with preoperative values.

\section{References}

1. Neurovascular compression (conflict)]. Slavik EE1, DjurovićBM et-al;ActaChirIugosl. 2008;55(2):161 2. Satoh T, Onoda K, Date I: Preoperative simulation for microvascular decompression in patients with idiopathic trigeminal neuralgia: Visualization with threedimensional magnetic resonance cisternogram and 
angiogram fusion imaging. Neurosurgery 60: 104-14, 2007

3. Satoh T, Onoda K, Date I: Preoperative simulation for microvascular decompression in patients with idiopathic trigeminal neuralgia: Visualization with threedimensional magnetic resonance cisternogram and angiogram fusion imaging. Neurosurgery 60: 104-14, 2007

4. Satoh T, Omi M, Nabeshima M, et al: Severity analysis of neurovascular contact in patients with trigeminal neuralgia: Assessment with the inner view of the $3 \mathrm{D} M R$ cisternogram and angiogram fusion imaging. AJNR Am J Neuroradiol 30: 603-7, 2009

5. Shojima M, Oshima M, Takagi K, et al: Magnitude and role of wall shear stress on cerebral aneurysm: computational fluid dynamic study of 20 middle cerebral artery aneurysms. Stroke 35: 2500-5, 2004

6. Toru SATOH, Keisuke ONODA et al: Local Hemodynamics of Compressed Blood Vessels in
Neurovascular Compression Site of Unilateral Facial Spasm Using Numerical Fluid Dynamics (CFD)

7. Janiga G, Sugiyama $K$, Kono $K$, et al: The computational fluid dynamics rupture challenge 2013Phase 1: Prediction of rupture status in intracranial aneurysms. AJNR Am J Neuroradiol 30:530-6, 2015

8. Hassan T, Timofeev EV, Saito T, et al: Proposed parent vessel geometry-based categorization of saccular intracranial aneurysms: computational flow dynamics analysis of the risk factors for lesion ruptur.JNeurosurg 103:662-80, 2005

9. Shojima M, Oshima M, Takagi K, et al: Magnitude and role of wall shear stress on cerebral aneurysm: computationalfluid dynamic study of 20 middle cerebral artery aneurysms. Stroke 35: 2500-5, 2004

10. Sforza DM, Putman CM, Tateshima S, et al: Effects of perianeurysmal environment during the growth of cerebral aneurysms: A case study. AJNR Am J Neuroradiol 3 :1115-20, 2012 